\title{
We Jean Garrabé (1931-2020)
}

Ce texte est issu d'un numéro spécial de la revue l'Evolution Psychiatrique d'hommage à Jean Garrabé

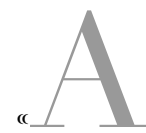

lors voyez vous, j’ai été étonné de lire que... » Ainsi Jean Garrabé s'adressait-il régulièrement à ses interlocuteurs. Évoquer ce prestigieux ami disparu cette année 2020 peut passer par divers canaux, sa bibliographie (considérable), son érudition (immense et éclectique), ses thèmes favoris (particulièrement variés), son rapport à l'histoire de notre discipline, etc. Mais également est-il possible de parler de l'homme pensant et de sa méthode dialogique qui l'entraînaient lui et son interlocuteur dans de vertigineux mais néanmoins rigoureux méandres dialectiques. Quand il étudiait un sujet, préparait un texte ou bien une conférence il semblait s'immerger totalement dans les références, les contradictions (contradictions qu'il savourait en gourmet du discours), en apnée, en abnégation. Alors l'interlocuteur, dont j'ai l'honneur d'avoir été, lui répondait quelque chose qui le laissait pensif. Et, si une nouvelle rencontre avait lieu quelques jours plus tard, et s'il n'avait pas terminé son travail, il s'adressait au même interlocuteur et commençait invariablement par “Alors voyez vous, j’ai été étonné de constater que... », et reprenait son argumentation à l'identique, la répétait. Il ne s'agissait nullement d'une attitude défensive érigeant un mur de savoir écrasant à des fins de domination, ni d'un radotage qui aurait oublié que cet échange avait déjà eu lieu dans les mêmes termes, mais d'une méthode, et l'on ne pouvait qu'être extrêmement fier lorsqu'un fragment de notre précédant échange apparaissait à la fin de son texte. Il s'agissait d'un exercice de maïeutique érigé comme méthode. Oui, texte, car il possédait un style textuel de dire, l'expression " parler comme un livre » lui correspondait parfaitement, de sa voix douce et monocorde il égrainait les mots avec lenteur faisant même sentir la ponctuation. Donc il reprenait, quelques jours plus tard, sans référence au fait qu'il prolongeait une conversation antérieure, ignorant l'interruption. Puis quand il en avait fini, quelques temps se passait et revenait l'insistant: “Alors voyez vous, j'ai été étonné... Un autre travail le happait et il happait du même coup ses interlocuteurs.

“ J'ai été étonné » revenait souvent dans sa conversation, étonnement presque juvénile mais aussi lucide et stratégique. Ainsi lors qu'untel soutenait une position inexcusable jamais de colère mais un étonnement attristé, et se disant étonné restait bien veillant mais implacable et tranquille. Cet étonnement constitutif faisait de lui un 
explorateur chronique du monde, de par ses nombreux voyages, de par ses lectures, de par ses rencontres. Mais aussi il riait tout en secousses face principalement aux paradoxes des institutions et des hommes, il avait l'humour des contradictions, à partir du moment où il s'agissait d'une bêtise honnête.

Jean n'était pas de ces tribuns solaires qui attrapent l'auditoire par des discours assez creux mais enrobés d'une atmosphère affective, théâtrale, spectaculaire : envers de l'histrion, parangon de la rigueur. Limites de la rigueur, sujet qui nous passionnait tous deux, quelle possibilité pour une rigueur de l'intuition lors d'une psychothérapie par exemple. Rien de mondain chez lui, au sens superficiel du terme, ni fausse chaleur feinte, ni connivence insincère, ni froideur non plus, mais juste distance, et rigueur toujours donc.

Avec Yves Thoret il avait été recruté à l'Évolution Psychiatrique du fait de ses qualités de psychiatre et d'auteur mais aussi afin de rajeunir l'effectif, de le renouveler, ce qu'il aimait à raconter, ce qui là encore le faisait sursauter de rire, lui qui avait l'âge d'être le père ou même le grand père de la plupart des membres de L'Évolution Psychiatrique. De même parlait il de Lacan qu'il croisait au comité de rédaction, de Henri Ey, et d'autres grands noms de la psychiatrie française ou internationale, racontait des anecdotes savoureuses et riait, toujours en secousses.

Pour en revenir à sa méthode, il était extrêmement prudent, n’induisait jamais d'hypothèse, ne formait aucun amalgame, tout au plus déduisait il sur la base de données fiables et concordantes, le plus souvent mettait en perspective les idées avec une démarche d'historien scrupuleux qui ne spéculait jamais : Il s'en tenait aux faits (J'ai été étonné...). Mais, et il n’en dérogeait jamais, aux faits remis dans leur contexte, historique, politique, culturel. Un jour que je discutais avec lui d'un propos de Ey qui fustigeait “ l'éclectisme » en psychiatrie il m'avait répondu qu'il fallait replacer cette phrase dans le débat de l'époque, et la conception unitaire de l'organodynamisme. En vérité principalement il était un grand sceptique et rien ne pouvait être ni généralisable ni définitif. Avec son ami Georges Lantéri-Laura il partageait l'idée simple que l'épistémologie de la psychiatrie était son histoire, que seule son histoire et sa contextualisation permettait de s'y retrouver et de se retrouver dans la psychiatrie actuelle, sinon l'errance guidait le psychiatre désarrimé. À ce propos la médecine de spécialité psychiatrique est la seule médecine à avoir autant besoin de son histoire pour penser sa clinique et ses concepts. On peut regretter à ce sujet le peu de cas qui est fait de l'histoire de notre discipline dans l'enseignement donné aux internes, et de les voir finir l'internant et “ faire des remplacements " dans des établissements exsangues. Nous sommes loin de l'engagement au long cour d'un Jean Garrabé, entre autre à l'institut Marcel Rivière où il été nommé par le Pr Paul Sivadon pour prendre sa suite en tant que médecin chef de l'institut, il y a développé la psychothérapie 
institutionnelle et une pratique imprégnée de psychanalyse. Comme à son habitude, quant Paul Sivadon lui avait proposé le poste, il avait été " étonné ", et avait posé la question : "Mais pourquoi moi ?" pour s'entendre répondre "parce que vous êtes ponctuel aux réunions ", cela je l'ai entendu raconter un nombre incalculable de fois et chaque fois son rire ponctuait et le secouait doucement. Je crois que plus il racontait cette anecdote plus il riait.

Il ne faudrait pas oublier le formidable clinicien qu'il était derrière l'érudit humaniste, ne perdant jamais de vue l'impossible dichotomie sujet-objet en psychothérapie, le psychothérapeute faisant partie intégrante du processus et n'étant d'aucune façon un praticien neutre observateur de l'évolution clinique de son patient. Le psychiatre soigne aussi la maladie qu'il produit notamment par le biais des prophéties autoréalisatrices construites par ses propres théories défensives (Les fameuses représailles théoriques)...

Cher Jean, je reste étonné de votre départ même si rationnellement je pouvais prévoir ce nouveau voyage. 